\title{
Epidemiology of hospital-acquired infections in cirrhotic patients: effect of carriage of methicillin-resistant Staphylococcus aureus and influence of previous antibiotic therapy and norfloxacin prophylaxis
}

\author{
B. CAMPILLO ${ }^{1 *}$, C. DUPEYRON ${ }^{2}$ AND J. P. RICHARDET ${ }^{1}$ \\ ${ }^{1}$ Service de Rééducation Digestive, Hôpital Albert Chenevier, 40 rue de Mesly, 94010 Créteil, France \\ ${ }^{2}$ Laboratoire Central, Hôpital Albert Chenevier, 40 rue de Mesly, 94010 Créteil, France
}

(Accepted 30 July 2001)

\section{SUMMARY}

We assessed the prevalence of carriage of methicillin-resistant Staphylococcus aureus (MRSA) in anterior nares and stools, and of third-generation cephalosporin resistant enterobacteriaceae and non-fermenting Gram-negative bacilli (RE/RNF) in stools of 748 hospitalized long-stay cirrhotic patients. We also evaluated the consequences of carriage on the epidemiology of hospital-acquired spontaneous bacterial peritonitis, bacteraemia and urinary tract infection (UTI) in these patients. The prevalence of carriage of MRSA and RE/RNF was $16.7 \%$ and $14.7 \%$ respectively. Whereas RE/RNF carriage did not lead to an increased risk of infection due to RE/RNF, the overall risk of infections caused by MRSA was more than tenfold higher in MRSA carriers. MRSA and RE/RNF carriers had received prior antibiotic therapy to a greater extent than non-carriers $(P<0 \cdot 001)$ and MRSA carriers had received prior norfloxacin prophylaxis to a greater extent than the two other groups $(P<0 \cdot 02)$. The mortality rate during hospital stay was higher in MRSA and RE/RNF carriers than in non-carriers $(P<0.001)$. Pugh score $(P<0 \cdot 0001)$, age $(P<0 \cdot 0001)$, MRSA carriage $(P=0.0018)$ and bacteraemia $(P=$ $0.0017)$ were associated independently with mortality. MRSA carriage in hospitalized cirrhotic patients leads to the emergence of infections due to this strain, mainly SBP and bacteraemia. Prior antibiotic therapy and norfloxacin prophylaxis increase the risk of carriage of MRSA.

\section{INTRODUCTION}

Bacterial infections in liver cirrhosis are an important cause of morbidity and mortality. The most frequent infections are spontaneous bacterial peritonitis (SBP), urinary tract infection (UTI), pneumonia, skin infections and bacteraemia [1], with Gram-negative bacteria, mainly enterobacteriaceae, predominating. These bacteria are derived from the intestinal Gram-negative flora, and the passage of viable bacteria from the gastrointestinal tract to extraintestinal sites seems to be an important step in the pathogenesis of SBP and other bacterial infections [2-6]. Little is known about hospital-acquired infections in cirrhosis and the risk of emergence of the

* Author for correspondence. resistant strains of bacteria that characterize nosocomial infections.

A high prevalence of carriage of methicillinresistant strains of Staphylococcus aureus (MRSA) has been reported in hospitalized cirrhotic patients suggesting a possible increased risk of MRSA infections. Many cirrhotic patients receive antibiotics for sepsis and norfloxacin prophylaxis decreases the risk of such infections due to Gram-negative bacteria but may facilitate the emergence of resistant Gramnegative bacteria and Gram-positive pathogens [7]. Thus, evaluation of the risk of emergence of infections caused by resistant bacterial strains seems important in patients with advanced liver disease who are exposed to frequent or long-term hospitalization.

In this prospective study, we investigated the 
epidemiology of hospital-acquired infections in a large population of cirrhotic patients. We assessed carriage of the most common bacterial strains involved in nosocomial infections, namely MRSA and third-generation cephalosporin resistant enterobacteriaceae and non-fermenting Gram-negative bacilli. We evaluated the consequences of carriage on the spectrum of bacteria causing infections developing during hospitalization. Our final aim was to assess the influence of previous antibiotic therapy and norfloxacin prophylaxis on carriage of these resistant bacterial strains.

\section{PATIENTS AND METHODS}

From 1 January 1996 to 30 January 2000, patients with liver cirrhosis admitted to our unit for chronic liver diseases were included in the study. Before admission to the unit, patients had been hospitalized for at least 2 weeks in another hospital for a complication of the disease such as severe malnutrition, sepsis, gastro-intestinal bleeding, refractory ascites or hepato-renal syndrome. Our unit is specially devoted to chronic liver disease and receives patients from many hospitals in the Paris area when long hospital stay is required because of the complications and severity of the disease. The diagnosis of cirrhosis was based on liver biopsy or conventional clinical, biological and endoscopic findings. The degree of liver failure was assessed by Child-Pugh classification (class A, well-preserved liver function; class B, mild liver failure; class $\mathrm{C}$, severe liver failure). Pugh scores are in the range 5-15 and increase as severity of liver failure worsens [8]. The aetiology of cirrhosis was alcoholism in $90 \%$ of cases, hepatitis $\mathrm{C}$ in $5 \%$ of cases (associated with alcoholism in $2 \%$ of cases), hepatitis $\mathrm{B}$ in $2.5 \%$ of cases and biliary or cryptogenic in the remaining cases.

The study included 748 patients who were screened within $48 \mathrm{~h}$ of admission for nasal carriage of Staphylococcus aureus, stool carriage of Staphylococcus aureus and Gram-negative bacilli resistant to third-generation cephalosporins including resistant enterobacteria and resistant non-fermenting bacilli (RE/RNF) and for urinary tract infection (UTI). None of the patients included in the study had a central venous catheter at the time of admission to our unit.

Nasal specimens (5 rotations in the 2 anterior nares) and stools (collected in small sterile containers) were sent to the bacteriology laboratory within $1 \mathrm{~h}$ of admission. Nasal swabs and stool samples were streaked onto Chapman agar to detect Staphylococcus aureus. Stool samples were also streaked onto two MacConkey agar plates supplemented respectively with cefotaxime $(0.5 \mu \mathrm{g} / \mathrm{ml})$ and ceftazidime $(2 \mu \mathrm{g} / \mathrm{ml})$ to detect RE/RNF. Media were incubated at $37^{\circ} \mathrm{C}$ for $48 \mathrm{~h}$. After morphological and microscopic examination, relevant bacteria were further identified.

Urine samples were collected as follows: the urethral meatus was washed with soap and rinsed with sterile water. The first morning urine was collected in sterile containers using a midstream clean-catch technique, and transported to the laboratory for urine analysis and culture no more than $1 \mathrm{~h}$ after collection. Urine samples were cultured by inoculating $0.05 \mathrm{ml}$ of $1 / 100$ diluted (in sterile water) specimen onto blood agar and MacConkey agar plates, with incubation at $37^{\circ} \mathrm{C}$ overnight. Colonies were counted and the organisms were identified.

If SBP and/or bacteraemia were suspected, blood and/or ascitic fluid was collected in blood-culture bottles at the bedside, incubated at $37^{\circ} \mathrm{C}$ for 7 days, and examined daily for turbidity. Bottles were subcultured after 2 and 7 days on chocolate-enriched agar plates for aerobic and anaerobic growth. Plates were incubated for 1 or 2 days at $37^{\circ} \mathrm{C}$, and organisms were identified.

All organisms were identified by their biochemical and immunological characteristics. A rapid slideagglutination test simultaneously detecting fibrinogen activity (clumping factor), protein $\mathrm{A}$ and capsular polysaccharides was used to identify Staphylococcus aureus (Pastorex Staph Plus, Sanofi Pasteur Diagnostics, Marne la Coquette, France), followed if necessary by the determination of free coagulase activity. Enterobacteriaceae and non fermenting bacilli were identified with API 20E and API 32GN strips, respectively (BioMérieux SA, 69 Marcy l'Etoile, France).

Antibiotic susceptibilities were determined using the disk diffusion technique, as recommended by the Antibiogram Committee of the French Society of Microbiology [9]. To detect methicillin resistance in Staphylococcus aureus, 5- $\mu \mathrm{g}$ oxacillin disks were used and plates were incubated for $24 \mathrm{~h}$ at $30{ }^{\circ} \mathrm{C}$. Antibiotic resistance patterns of all isolates were studied and compared.

Staphylococcal carriage was defined as the presence of Staphylococcus aureus in nasal and/or stool cultures. Antimicrobial susceptibility testing was used 
to distinguish methicillin-sensitive (MSSA) and methicillin-resistant (MRSA) strains. RE/RNF stool carriage was defined by the presence of Gram-negative bacilli resistant to third-generation cephalosporins (enterobacteria or non-fermenting bacilli) in stools, as determined by culture on cefotaxime and ceftazidimesupplemented media; each strain detected on one or both supplemented medium was taken into account. Resistance was confirmed by further antimicrobial susceptibility testing.

All episodes of UTI, SBP and bacteraemia with positive culture occurring during the hospital stay in our unit were analysed.

UTI was defined by a leucocyte count exceeding $10000 / \mathrm{ml}$ and bacteriuria, according the criteria of Kass, and regardless of clinical signs [10]. Patients with urinary catheters were excluded from the study.

The diagnosis of SBP was based on the combination of a positive ascitic fluid culture and a polymorphonuclear cell count of $>250 \mathrm{~mm}^{3}$, irrespective of clinical signs of SBP, or a positive ascitic fluid culture with no increase in the polymorphonuclear cell count but with abdominal pain and fever. This symptomatic form of bacterial ascites is considered to be a variant of SBP [11-15]. The diagnosis of bacteraemia was based on a combination of clinical signs (fever, hypothermia, encephalopathy and hypotension) and one or several positive blood cultures. Contaminants in ascitic fluid and blood cultures were excluded.

Quantitative variables are given as the mean \pm s.D. Comparison of variables among three groups of patients were performed using one-way analysis of variance (ANOVA). When ANOVA showed a significant difference, two groups were compared with Bonferroni-Dunn's test. Comparisons of percentages between groups were made with the $\chi^{2}$ test, with Yates' correction when required. Multivariate analysis was done using a stepwise logistic regression model. The threshold for statistical differences was $P<0.05$. Data were analysed with Epi-Info software VS (CDC Atlanta) and Statview software.

\section{RESULTS}

Of the 748 patients studied, 125 were MRSA carriers on admission to the unit. MRSA carriage was found in stools and nose in 68 cases, in stools only in 25 cases and in nose only in 32 cases. The overall prevalence of MRSA carriage on admission was $16.7 \%$. Of these 125 patients, 18 also carried RE/RNF in stools. Isolated carriage of RE/RNF in stools was found in a further 92 patients who made up the group of $\mathrm{RE} / \mathrm{RNF}$ carriers. The overall prevalence of RE/ RNF carriage was $14.7 \%$. Numbers of patients colonized with various $\mathrm{RE} / \mathrm{RNF}$ strains were as follows: Enterobacter cloacae 40, Citrobacter freundii 27, Pseudomonas aeruginosa 19, Acinetobacter baumanii 7, Enterobacter aerogenes 5, Pseudomonas species 4, Stenotrophomonas maltophila 4, Klebsiella pneumoniae 4, Escherichia coli 3, Hafnia alvei 3, Klebsiella oxytoca 2 and Enterobacter sakasaki 1. Two different strains of RE/RNF were isolated from the stools of 9 patients and one strain only from each of the remaining patients.

Carriage of MRSA and RE/RNF in relation to Child classification, mean patient age, Pugh score and duration of hospital stay is shown in Table 1. MRSA carriage was higher in Child $\mathrm{C}$ patients but the difference was not significant. Age, Pugh score and duration of hospital stay were significantly different among the three groups of patients. When compared with non-carrier patients, RE/RNF carriers were older $(P<0.03)$ while MRSA carriers had a higher Pugh score and a longer duration of hospital stay in the unit $(P<0 \cdot 01)$.

Of the 748 patients studied, 173 were treated with antibiotics ( $\beta$-lactams, third generation cephalosporins, fluoroquinolones) for at least 10 days for sepsis and additionally, 183 patients were given norfloxacin for primary or secondary prophylaxis of SBP for at least 2 weeks before admission to the unit. If being given norfloxacin prophylaxis, patients took antibiotics until admission, then norfloxacin was withdrawn. The numbers and percentages of carrier and non-carrier patients receiving prior antibiotic therapy or norfloxacin prophylaxis are shown in Table 2. In comparison with non-carrier patients, MRSA and RE/RNF carriage was more common in patients who had received prior antibiotic therapy $(P<0.001)$. A greater proportion of MRSA carriers had received norfloxacin prophylaxis than non-carrier patients $(P<0.05)$, but there was no significant difference between RE/RNF carriers and non-carrier patients. Among the 43 MRSA carriers who had had previous norfloxacin prophylaxis, MRSA carriage was found in nose and stools in 27 cases, in stools only in 7 cases and in the nose only in 9 cases.

Sixty-three episodes of SBP, 45 episodes of bacteraemia and 114 episodes of UTI occurred in 60, 45 and 99 non-carrier patients respectively. Twentyeight episodes of SBP, 30 episodes of bacteraemia and 50 episodes of UTI occurred respectively in 19, 22 and 
Table 1. Carriers of MRSA and RE/RNF according to Child classification

\begin{tabular}{lllll}
\hline \hline & MRSA carriers & RE/RNF carriers & Non-carriers & Total \\
\hline Child A & $9(7 \cdot 2)$ & $7(7 \cdot 6)$ & $73(13 \cdot 7)$ & $89(11 \cdot 9)$ \\
Child B & $45(36 \cdot 0)$ & $36(39 \cdot 1)$ & $200(37 \cdot 7)$ & $281(37 \cdot 6)$ \\
Child C & $71(56 \cdot 8)$ & $49(53 \cdot 3)$ & $258(48 \cdot 6)$ & $378(50 \cdot 5)$ \\
Total & 125 & 92 & 531 & 748 \\
M/F & $85 / 40$ & $61 / 31$ & $335 / 196$ & $481 / 267$ \\
Age (years) & $55 \cdot 8 \pm 11 \cdot 4$ & $56 \cdot 7 \pm 11 \cdot 6^{*}$ & $53 \cdot 8 \pm 11 \cdot 7 * \dagger$ & $54 \cdot 5 \pm 11 \cdot 7$ \\
PUGH score & $9 \cdot 9 \pm 2 \cdot 1^{* *}$ & $9 \cdot 8 \pm 2 \cdot 2$ & $9 \cdot 3 \pm 2 \cdot 4^{* *+\dagger \dagger}$ & $9 \cdot 5 \pm 2 \cdot 3$ \\
Hospital stay (days) & $43 \cdot 4 \pm 39 \cdot 6^{* *}$ & $40 \cdot 3 \pm 28 \cdot 8$ & $35 \cdot 2 \pm 23 \cdot 0^{* *+\dagger \dagger}$ & $37 \cdot 1 \pm 27 \cdot 4$ \\
\hline \hline
\end{tabular}

Means are given \pm S.D. Percentages are in parentheses.

$* P<0.03, * * P<0.01$ (Bonferroni-Dunn's test).

$\dagger P<0.05, \dagger \dagger P<0.02, \dagger \dagger P<0.01$ (ANOVA).

Table 2. Prevalence of patients receiving prior antibiotic therapy or norfloxacin prophylaxis in carrier and noncarrier groups

\begin{tabular}{|c|c|c|c|c|c|}
\hline & $\begin{array}{l}\text { MRSA carriers } \\
(n=125)\end{array}$ & $\begin{array}{l}\text { RE/RNF carriers } \\
(n=92)\end{array}$ & $\begin{array}{l}\text { Non-carriers } \\
(n=531)\end{array}$ & $\begin{array}{l}\text { Total } \\
(n=748)\end{array}$ & \\
\hline $\begin{array}{l}\text { Patients with previous } \\
\text { antibiotic therapy }\end{array}$ & $57(45 \cdot 6)$ & $43(46 \cdot 7)$ & $73(13 \cdot 7)$ & 173 & $P<0.001$ \\
\hline $\begin{array}{l}\text { Patients with previous } \\
\text { norfloxacin prophylaxis }\end{array}$ & $43(34 \cdot 4)$ & $22(23 \cdot 9)$ & $118(22 \cdot 2)$ & 183 & $P<0.02$ \\
\hline
\end{tabular}

Percentages are given in parentheses.

Table 3. Prevalence of septic complications in carrier and non-carrier groups

\begin{tabular}{llllll}
\hline \hline & $\begin{array}{l}\text { MRSA carriers } \\
(n=125)\end{array}$ & $\begin{array}{l}\text { RE/RNF carriers } \\
(n=92)\end{array}$ & $\begin{array}{l}\text { Non-carriers } \\
(n=531)\end{array}$ & $\begin{array}{l}\text { Total } \\
(n=748)\end{array}$ & \\
\hline SBP* & $19(15 \cdot 2)$ & $14(15 \cdot 2)$ & $60(11 \cdot 3)$ & 93 & n.s. \\
Bacteraemia & $22(17 \cdot 6)$ & $8(8 \cdot 7)$ & $45(8 \cdot 5)$ & 75 & $P<0 \cdot 01$ \\
UTI $\dagger$ & $34(27 \cdot 2)$ & $26(28 \cdot 3)$ & $99(18 \cdot 6)$ & 159 & $P<0 \cdot 01$ \\
\hline \hline
\end{tabular}

Percentages are given in parentheses.

* SBP, spontaneous bacterial peritonitis; †UTI, urinary tract infection.

34 MRSA carriers. Fifteen episodes of SBP, 8 episodes of bacteraemia and 30 episodes of UTI occurred respectively in 14, 8 and $26 \mathrm{RE} / \mathrm{RNF}$ carriers.

The numbers and percentages of patients with septic complications in carrier and non-carrier groups are shown in Table 3. The percentage of patients with SBP was higher in MRSA and RE/RNF carriers but the difference was not significant. Bacteraemia was more frequent in MRSA carriers than in non-carriers $(P<0 \cdot 01)$, but there was no significant difference between bacteraemia rates in RE/RNF carriers and non-carrier patients. UTI was more frequent in both MRSA and RE/RNF carriers, in comparison with non-carriers $(P<0 \cdot 01)$.

The nature of the bacteria responsible for SBP, bacteraemia and UTI in the three groups of patients is shown in Table 4. Enterobacteriaceae were isolated with a lower rate in bacteraemia and UTI in MRSA carriers in comparison with the two other groups (respectively $3.3 \%$ vs. $28.9 \%$ and $33.3 \%$ for bacteraemia, $P<0.05 ; 46.0 \%$ vs. $71.9 \%$ and $80.0 \%$ for UTI, $P<0.01)$. Conversely, infection with MRSA was found with a higher rate in MRSA carriers in SBP (46.4\% vs. $11 \cdot 1 \%$ and $0 \%, P<0.001)$, bacteraemia $(53.3 \%$ vs. $6.7 \%$ and $0 \%, P<0.001)$, UTI $(24.0 \%$ vs. $4.4 \%$ and $0 \%, P<0.001)$. Over four fifths $(56 / 68$, $82.4 \%$ ) of Staphylococcus aureus strains isolated in the entire population were MRSA. The prevalence of total Gram-positive pathogens was comparable in the three groups for SBP and bacteraemia but was higher 
Table 4. Bacteria cultured from ascitic fluid, blood culture and urine in carriers and non-carriers

\begin{tabular}{|c|c|c|c|c|c|c|c|c|c|}
\hline & \multicolumn{3}{|c|}{ Non-carrier $\dagger$} & \multicolumn{3}{|c|}{ MRSA carriers } & \multicolumn{3}{|c|}{$\mathrm{RE} / \mathrm{RNF}$ carrier } \\
\hline & A & B & $\mathrm{U}$ & A & B & $\mathrm{U}$ & A & B & $\mathrm{U}$ \\
\hline Escherichia coli & 13 & 11 & 60 & 3 & & 13 & 6 & 2 & 10 \\
\hline Other Enterobacteriaceae & 7 & 2 & 22 & 2 & 1 & 10 & 1 & & 14 \\
\hline Total Enterobacteriaceae & $\begin{array}{l}20 \\
(31 \cdot 7 \%)\end{array}$ & $\begin{array}{l}13 \\
(28 \cdot 9 \%)^{\mathrm{a}}\end{array}$ & $\begin{array}{l}82 \\
(71.9 \%)^{b}\end{array}$ & $\begin{array}{l}5 \\
(17 \cdot 9 \%)\end{array}$ & $\begin{array}{l}1 \\
(3 \cdot 3 \%)^{\mathrm{a}}\end{array}$ & $\begin{array}{l}23 \\
(46 \cdot 0 \%)^{b}\end{array}$ & $\begin{array}{l}7 \\
(46 \cdot 7 \%)\end{array}$ & $\begin{array}{l}2 \\
(33 \cdot 3 \%)^{\mathrm{a}}\end{array}$ & $\begin{array}{l}24 \\
(80 \cdot 0 \%)^{b}\end{array}$ \\
\hline $\begin{array}{l}\text { Non-fermenting } \\
\text { Gram-negative bacilli }\end{array}$ & 3 & 2 & 6 & 2 & 1 & 4 & & & 3 \\
\hline Bacteroides spp. & 1 & & & & 1 & & & & \\
\hline Streptococcus spp. & 17 & 9 & 2 & 6 & 4 & 1 & 3 & 1 & 1 \\
\hline Enterococcus spp. & 12 & 2 & 11 & 2 & 1 & 8 & 4 & 1 & 2 \\
\hline MRSA & $\begin{array}{l}7 \\
(11 \cdot 1 \%) \\
*\end{array}$ & $\begin{array}{l}3 \\
(6 \cdot 7 \%) \\
* *\end{array}$ & $\begin{array}{l}5 \\
(4 \cdot 4 \%) \\
* * *\end{array}$ & $\begin{array}{l}13 \\
(46 \cdot 4 \%) \\
*\end{array}$ & $\begin{array}{l}16 \\
(53 \cdot 3 \%) \\
* *\end{array}$ & $\begin{array}{l}12 \\
(24 \cdot 0 \%) \\
* * *\end{array}$ & $\begin{array}{l}(0 \%) \\
*\end{array}$ & $\begin{array}{l}(0 \%) \\
* *\end{array}$ & $\begin{array}{l}(0 \%) \\
* * *\end{array}$ \\
\hline MSSA & 1 & 5 & 5 & & & 1 & & & \\
\hline $\begin{array}{l}\text { Coagulase negative } \\
\text { staphylococcus }\end{array}$ & 1 & 11 & 2 & & 6 & & & 4 & \\
\hline Clostridium spp. & 1 & & & & & & 1 & & \\
\hline $\begin{array}{l}\text { Total Gram-positive } \\
\text { pathogens } \\
\text { Candida albicans }\end{array}$ & $\begin{array}{l}39 \\
(61 \cdot 9 \%)\end{array}$ & $\begin{array}{l}30 \\
(66 \cdot 7 \%)\end{array}$ & $\begin{array}{l}25 \\
(21 \cdot 9 \%)^{\mathrm{c}} \\
1\end{array}$ & $\begin{array}{l}21 \\
(75 \%)\end{array}$ & $\begin{array}{l}27 \\
(90 \cdot 0 \%)\end{array}$ & $\begin{array}{l}22 \\
(44 \%)^{\mathrm{c}} \\
1\end{array}$ & $\begin{array}{l}8 \\
(53 \cdot 9 \%)\end{array}$ & $\begin{array}{l}6 \\
(66 \cdot 6 \%)\end{array}$ & $\begin{array}{l}3 \\
(10 \%)^{\mathrm{e}}\end{array}$ \\
\hline Total & 63 & 45 & 114 & 28 & 30 & 50 & 15 & 8 & 30 \\
\hline
\end{tabular}

$\uparrow$ A, Ascites; B, blood; U, urine.

${ }^{\mathrm{a}} P<0.05 ;{ }^{\mathrm{b}, \mathrm{c}} P<0.01 ; *,{ }^{* *},{ }^{* * *} P<0 \cdot 001$.

Table 5. Risk of MRSA infection in carriers and non-carriers

\begin{tabular}{lllll}
\hline \hline & $\begin{array}{l}\text { MRSA carriers } \\
(n=125)\end{array}$ & $\begin{array}{l}\text { RE/RNF carriers } \\
(n=92)\end{array}$ & $\begin{array}{l}\text { Non-carriers } \\
(n=531)\end{array}$ & \\
\hline SBP* & $13(10 \cdot 4)$ & $0(0)$ & $7(1 \cdot 3)$ & $P<0 \cdot 001$ \\
Bacteraemia & $16(12 \cdot 8)$ & $0(0)$ & $3(0 \cdot 6)$ & $P<0 \cdot 001$ \\
UTI* & $12(9 \cdot 6)$ & $0(0)$ & $5(0 \cdot 9)$ & $P<0 \cdot 001$ \\
Total & $41(32 \cdot 8)$ & $0(0)$ & $15(2 \cdot 8)$ & $P<0 \cdot 001$ \\
\hline \hline
\end{tabular}

Percentages are given in parentheses.

* See footnote, Table 3.

in MRSA carriers in UTI $(44.0 \%$ vs. $21.9 \%$ and $10 \cdot 0 \%, P<0 \cdot 01)$.

In MRSA carriers, MRSA strains were isolated from ascitic fluid, blood culture and urine after a mean interval following admission of $16 \pm 26$ days (extremes: 0-120 days, median: 13 days). The risk of infections related to MRSA in the three groups of patients is shown in Table 5. MRSA carriers had an increased risk of SBP, bacteraemia and UTI caused by MRSA in comparison with the two other groups, the overall risk was more than tenfold higher than in noncarriers.

Three patients carrying a resistant enterobacterial strain had episodes of infection caused by the same species and with the same antibiotic resistance pattern (UTI - two cases, bacteraemia - one case, Citrobacter freundii in one case, Enterobacter cloacae in two cases). Carriage and infection coincided in two cases.

The mortality rate during hospital stay was higher in MRSA carriers $(29 \cdot 6 \%, 37 / 125)$ and in RE/RNF carriers $(25.0 \%, 23 / 92)$ than in non-carrier patients $(14.1 \%, 75 / 531)(P<0 \cdot 001)$. A septic complication was the direct cause of death in $45 \%$ of MRSA carriers, $47.8 \%$ of RE/RNF carriers and $34.7 \%$ of non-carrier patients (n.s.). The cause of death was liver failure in the remaining patients. Pugh score and age at admission were higher in patients who died during hospital stay than in surviving patients 
(respectively $11 \cdot 1 \pm 1 \cdot 8 \quad$ vs. $9 \cdot 1 \pm 2 \cdot 3, \quad P<0 \cdot 001$; $60 \cdot 8 \pm 10 \cdot 9$ vs. $53 \cdot 2 \pm 11 \cdot 4$ years, $P<0 \cdot 001)$. In multivariate analysis with mortality as the dependent variable, Pugh score (OR: $1.669 \mathrm{CI}_{95}$ 1.485-1.876, $P<0.0001)$, age (OR: $1.083 \quad \mathrm{CI}_{95} \quad 1 \cdot 060-1 \cdot 105$, $P<0 \cdot 0001$ ), MRSA carriage (OR: $2 \cdot 305, \mathrm{CI}_{95} 1 \cdot 363-$ $3.897, P=0 \cdot 0018)$ and bacteraemia (OR: $2 \cdot 99, \mathrm{CI}_{95}$ $1.507-5.918, P=0.0017)$ were associated with mortality.

\section{DISCUSSION}

In this study series of cirrhotic patients, we chose for practical reasons to detect MRSA carriage on admission only. We showed that carriage is a strong predictor of subsequent infections caused by this strain. We were also able to document the prevalence of the different micro-organisms involved in hospitalacquired SBP, bacteraemia and ITU in cirrhotic patients, since all the patients included in the study were hospitalized before admission to our unit and were then exposed to a long hospital stay. Enterobacteriaceae and especially Escherichia coli have been reported to be the main agents responsible for SBP [15], but these data refer principally to communityacquired infections. The prevalence of different bacteria involved in hospital-acquired infections in cirrhotic patients has not been extensively studied as yet. We have shown previously changes in the nature of bacteria causing SBP over a 20 year period with a decrease in the prevalence of Enterobacteriaceae from $78.7 \%$ to $60.3 \%$. Moreover, resistance of bacteria increases as shown by the emergence of enterobacterial strains resistant to third generation cephalosporins since 1993 [16]. In this study, of a total of 106 episodes of SBP (in 93 patients) the overall prevalence of Enterobacteriaceae was only $30 \cdot 2 \%$ with a prevalence of $20.8 \%$ for Escherichia coli. In contrast, the prevalences of Streptococcus spp., Enterococcus spp. and Staphylococcus spp. were respectively $24 \cdot 5,17 \cdot 0$ and $19.8 \%$. We found a high prevalence of Staphylococcus aureus in SBP, with most of the strains isolated being methicillin resistant. Staphylococcus spp. predominated in bacteraemia, again with a high prevalence of MRSA. Conversely, Enterobacteriaceae predominated in UTI, with a prevalence of $66.5 \%$, compared with a prevalence of Staphylococcus aureus of $11 \cdot 3 \%$. Most Staphylococcus aureus strains were MRSA. We found a high prevalence of Staphylococcus aureus, mainly MRSA, whatever the site of infection. Carriage of MRSA at time of admission was the main contributory factor.

Today, MRSA is recognized as a major nosocomial pathogen, causing nosocomial infections in community and referral hospitals, as well as in long-term care facilities throughout the world [17, 18]. Two previous studies have assessed the prevalence of carriage of staphylococcal strains in cirrhotic patients, but only by sampling of the anterior nares and without sampling stools. Chapoutot et al. found a prevalence of MRSA carriage of $5.8 \%$ on admission while Chang et al. found a prevalence of $16.6 \%$ [19, 20]. The latter result was very close to that observed in our study $(16 \cdot 7 \%)$. The ecological niche of staphylococci is the anterior nares and previous studies have shown that the nares are the most consistent site from which the organism can be isolated [18]. We have also studied the carriage in stools and we have shown that though both sites are colonized in most cases (54\%), the organism is isolated from stools only in $20 \%$ of cases, demonstrating that assessing nasal carriage only may underestimate the true prevalence of carriage.

Several factors may account for this high prevalence of carriage. All these patients had been exposed to long prior hospital stay since they were inpatients before admission to our unit. Many had end-stage liver diseases with a high risk of septic complications and extensive prior use of antibiotic therapy. Little is known about the risk of infection related to this organism in carrier cirrhotic patients. We found an increased prevalence of MRSA strains not only in bacteraemia as expected but also in SBP and UTI in carrier patients. The presence of MRSA in anterior nares and stools suggests that multiple sites may often be colonized, facilitating episodes of bacteraemia. In this study, nasal and stool culture were performed only on admission and were not repeated later during the hospital stay. It is therefore possible that the prevalence of carriage increased during hospitalization, which may account for the 15 cases of infection due to MRSA that were observed in the non-carrier patients.

Infections are a major cause of morbidity and a significant contributor to death in patients with cirrhosis. The mortality rate was high in our patient population including many patients with end-stage liver disease. One third to one half of deaths were directly caused by sepsis and we found that bacteraemia was a independent predicator of mortality. MRSA carriers had more advanced disease 
liver disease, while RE/RNF carriers were older than non-carriers, which may account for their higher mortality rate. However MRSA carriage was an independent predictor of mortality, and the higher prevalence of bacteraemia in MRSA carriers is likely to be a contributory factor to the adverse prognostic impact of MRSA carriage.

In our study MRSA carrier patients had received prior antibiotic therapy to a greater extent than noncarrier patients [17]. As expected, previous antibiotic therapy had been also given to a greater extent in RE/RNF carrier patients. Norfloxacin is a widely used drug for long term selective intestinal decontamination in cirrhotic patients because it is incompletely absorbed from the intestine, is highly active against Gram-negative bacilli, has low activity against anaerobic bacteria and reduces dramatically the risk of infections carried by Gram-negative bacilli [21-23]. We showed in a previous study that long-term norfloxacin administration carries a risk of disturbing the bacterial ecology of patients with emergence of enterobacterial strains highly resistant to quinolones and the occurrence of Staphylococcus aureus and Staphylococcus spp. in the stools [24]. All the staphylococcal strains were methicillin resistant. More recently, we have shown that long-term norfloxacin administration in cirrhotic patients promotes staphylococcal SBP and bacteraemia and increases the risk of carriage of the methicillin resistant phenotype [7]. Because of this risk of emergence, we have chosen to stop administering prophylactic norfloxacin to patients admitted to our unit. In this study, we have shown that norfloxacin is a risk factor for carriage of MRSA. MRSA was found in stools in carrier patients taking norfloxacin, suggesting that changes in intestinal bacterial ecology may have a role in colonization. These data suggest that the increased risk of staphylococcal infections due to administration of norfloxacin is related to carriage of this strain. Regarding the different patterns of resistance to quinolone in different pathogens, the most striking has been the rapid emergence of a high prevalence of quinolone resistance $(>90 \%)$ among methicillinresistant but not methicillin-susceptible strains of Staphylococcus aureus in many parts of the world [25].

We also found a high prevalence of carriage of RE/RNF strains $(14.7 \%)$ in patients exposed to a long hospital stay. However, unlike MRSA carriage, $\mathrm{RE} / \mathrm{RNF}$ carriage was not associated with an increased risk of infection caused by RE/RNF strains since only three cases of infection caused by the same
RE strain which was found in the stools were observed. Norfloxacin prophylaxis was not given to a greater extent in RE/RNF carrier patients than in non-carrier patients showing that norfloxacin prophylaxis does not seem to increase the risk of carrying these strains; this finding is in agreement with studies showing that Escherichia coli resistant to norfloxaxin isolated in patients receiving this antibiotic are not resistant to third-generation cephalosporin [26].

In conclusion, we have shown that cirrhotic patients with advanced disease necessitating long hospital stay have a high prevalence of MRSA carriage which is a main factor predisposing to infections caused by this bacterial strain. Thus, MRSA carriage has important clinical consequences and is a poor prognostic factor as shown by the higher mortality rate of carrier patients. Staphylococcal infections contribute in greater part to the epidemiology of infections in cirrhotic inpatients when compared to that observed in community infections. Previous antibiotic therapy and norfloxacin prophylaxis increases the risk of MRSA carriage. Carriage should be assessed in patients submitted to frequent or long term hospitalization because approaches to the elimination of carriage based on disinfectants and local antibiotics are available. Also, use of norfloxacin should be limited in these patients because of the risk of emergence of MRSA infections [27].

\section{REFERENCES}

1. Caly WR, Strauss E. A prospective study of bacterial infections in patients with cirrhosis. J Hepatol 1993; 18: 353-8.

2. Guarner C, Soriano G. Spontaneous bacterial peritonitis. Semin Liver Dis 1997; 17: 203-17.

3. Runyon BA. Bacterial infections in patients with cirrhosis. J Hepatol 1993; 18: 271-2.

4. Runyon BA, Squier S, Borzio M. Translocation of gut bacteria in rats with cirrhosis to mesenteric lymph nodes partially explains the pathogenesis of spontaneous bacterial peritonitis. J Hepatol 1994; 21 : 792-6.

5. Garcia-Tsao B, Albillos A, Barden GE, West AB. Bacterial translocation in acute and chronic portal hypertension. Hepatology 1993; 17: 1081-5.

6. Llovet JM, Bartoli R, Planas R, et al. Bacterial translocation in cirrhotic rats. Its role in the development of spontaneous bacterial peritonitis. Gut 1994; 35: 1648-52.

7. Campillo B, Dupeyron C, Richardet JP, Mangeney N, Leluan G. Epidemiology of severe hospital-acquired infections in patients with liver cirrhosis: effect of longterm administration of norfloxacin. Clin Infect Dis 1998; 26: 1066-70. 
8. Pugh RNH, Murray-Lyon IM, Dawson JL, et al. Transsection of the œsophagus for bleeding oesophageal varices. Br J Surg 1973; 60: 646-9.

9. Comité de l'antibiogramme de la Société Française de Microbiologie. Communiqué, 1998. Pathol Biol 1998; 46: $1-16$.

10. Kass EH. Bacteriuria and the diagnosis of infections of the urinary tract. Arch Intern Med 1957; 100: 709-13.

11. Runyon BA. Spontaneous bacterial peritonitis: an explosion of information. Hepatology 1988; 8: 171-5.

12. Bar-Meir S, Lerner R, Conn NO. Analysis of ascitic fluid in cirrhosis. Dig Dis Sci 1979; 24: 136-44.

13. Pelletier G, Lesiu G, Ink O, et al. Asymptomatic bacterascites: is it spontaneous bacterial peritonitis? Hepatology 1991; 14: 112-5.

14. Chu CM, Chang KY, Lian YF. Prevalence and prognostic significance of bacterascites in cirrhosis with ascites. Dig Dis Sci 1995; 40: 561-5.

15. Rimola A, Garcia-Tsao G, Navasa M, et al. Diagnosis, treatment and prophylaxis of spontaneous bacterial peritonitis: a consensus document. J Hepatol 2000; 32 : 142-53.

16. Dupeyron C, Campillo B, Mangeney N, Richardet JP, Leluan G. Changes in nature and antibiotic resistance of bacteria causing peritonitis in cirrhotic patients over a 20 year period. J Clin Pathol 1998; 51: 614-6.

17. Voss A, Doebbeling BN. The worldwide prevalence of methicillin-resistant Staphylococcus aureus. Int J Antimicrob Agents 1995; 5: 101-6.

18. Kluytmans J, Van Belkum A, Verbrugh H. Nasal carriage of Staphylococcus aureus: epidemiology, underlying mechanisms, and associated risks. Clin Microbiol Rev 1997; 10: 505-10.

19. Chapoutot C, Pageaux GP, Perrigault PF, et al. Staphylococcus aureus nasal carriage in 104 cirrhotic and control patients. A prospective study. J Hepatol 1999; 30: 249-53.

20. Chang FY, Singh N, Gayowski T, Wagener MM, Marino IR. Staphylococcus aureus nasal colonization in patients with cirrhosis: prospective assessment of association with infection. Infect Control Hosp Epidemiol 1998; 19: 328-32.

21. Gines P, Rimola A, Planas R, et al. Norfloxacin prevents spontaneous bacterial peritonitis recurrence in cirrhosis: results of a double-blind, placebo-controlled trial. Hepatology $1990 ; 12$ : 716-24.

22. Soriano G, Guarner C, Teixedo M, et al. Selective intestinal decontamination prevents spontaneous bacterial peritonitis. Gastroenterology 1991; 100: 477-81.

23. Grange JD, Roulot D, Pelletier G, et al. Norfloxacin primary prophylaxis of bacterial infections in cirrhotic patients with ascites: a double-blind randomized trial. J Hepatol 1998; 29: 430-6.

24. Dupeyron C, Mangeney N, Sedrati L, Campillo B, Fouet P, Leluan G. Rapid emergence of quinolone resistance in cirrhotic patients treated with norfloxacin to prevent spontaneous bacterial peritonitis. Antimicrob Agents Chemother 1994; 38: 340-4.

25. Hopper DC. New uses for new and old quinolones and the challenge of resistance. Clin Infect Dis 2000; 30: 243-54.

26. Ortiz J, Vila MC, Soriano G, et al. Infections caused by Escherichia coli resistant to norfloxacin in hospitalized cirrhotic patients. Hepatology 1999; 29: 1064-9.

27. Reagan DR, Doebbeling BN, Pfaller MA, et al. Elimination of coincident Staphylococcus aureus nasal and hand carriage with intranasal application of mupirocin calcium ointment. Ann Intern Med 1991; 114: 101-6. 